\title{
Politik in Demokratien ohne demokratischen Souverån
}

\section{Das Scheitern der demokratischen Konsolidierung in Mittelamerika 1}

Als in den 80er Jahren die Militärregimes im südlichen Amerika zusammenbrachen, wurde auch in Mittelamerika - wenngleich aus anderen Motiven - die Stunde der Demokratie eingeläutet. Das Militär zog sich von der politischen Bühne zurück und die mittelamerikanischen Übergänge zur Demokratie werden seitdem allgemein als gelungen angesehen. Von einer Konsolidierung der jungen Demokratien kann aber immer noch keine Rede sein. Breite Bevölkerungskreise scheinen die neuen Regimes als Fremdkörper wahrzunehmen, der auf Desinteresse und sogar Ablehnung stößt. Die in den Transitionen eingeführten institutionellen und normativen Requisiten der Demokratie korrespondieren kaum mit den politischen Artikulationsformen, die sich in den jeweiligen Gesellschaften außerhalb des staatlich-institutionellen Rahmens traditionell herausgebildet haben. Die Artikulation eines erheblichen Teils gesellschaftlicher Kollektivinteressen findet in mehr oder weniger unmittelbaren politischen Handlungsformen mit stark kollektiv-partizipatorischen Elementen statt.

Die demgegenüber auf zentralstaatlicher Ebene implementierte symbolische Form politischen Handelns und die damit einhergehende Beschränkung politischer Partizipation auf den Wahlakt führt kaum zu einer politischen Integration, sie festigt eher den Ausschluß von der politischen Entscheidungssphäre. Der aufgrund seiner wirtschaftlich und sozial prekären Lage ohnehin geschwächte demokratische Souverän sieht sich dadurch um seine politischen Einflußmöglichkeiten gebracht.

In Mittelamerika stehen sich unterschiedliche Repräsentationsfiguren gegenüber, zwischen denen bisher keine Vermittlung existiert. Die demokratischen Regimes erweisen sich als unangepaßt und konsolidierungsunfähig,

1 Dieser Text ist im Zusammenhang mit dem Forschungsprojekt $»$ Die Demokratisierungen in Mittelamerika und die Einbeziehung der unteren Bevölkerungsschichten« entstanden (vgl. Tangermann/Rios 1994 und Tangermann 1995), das von 1992-95 in Nicaragua durchgeführt und von BUNTSTIFT gefördert wurde. 
da sie sich nicht auf die politischen Repräsentationsformen der gesellschaftlichen Interessen beziehen.

\section{Demokratisierung in Mittelamerilka ${ }^{2}$}

Nachdem die Sandinisten in Nicaragua 1979 die Diktatur der Somozas beseitigt und ein revolutionäres Regime errichtet hatten, verschwanden $a b$ Anfang der 80er Jahre die Militärregimes in der ganzen Region. Die nahezu zeitgleiche Einführung der Demokratie in Guatemala, Honduras und El Salvador hatte nicht zuletzt externe Gründe. Von der damaligen USRegierung unter Präsident Reagan wurde das Regime der Sandinisten als eine Bedrohung für die Stabilität in der Region angesehen, und so initiierte die US-Regierung - ähnlich wie sie es nach der kubanischen Revolution mit der kontinentalen »Allianz für den Fortschritt« getan hatte - eine regionale Anstrengung für mehr Demokratie und erreichte in den drei Ländern den baldigen Rückzug der Militärs von der politischen Bühne. ${ }^{3}$

Nach der Wahlniederlage der Sandinisten 1990 wurde in Nicaragua ein ähnliches demokratisches Regime errichtet wie in den Nachbarländern. Damit bestehen seit 1990 in ganz Mittelamerika liberale Demokratien. Obwohl alle Länder über gleiche politische Regimes verfügen, ist der Zustand dieser Demokratien sehr unterschiedlich. Die vier (relativ) neuen Demokratien, Guatemala, Honduras, El Salvador und Nicaragua, gehören

2 Mit Mittelamerika sind hier im traditionellen Verständnis die fünf Länder Guatemala, Honduras, El Salvador, Nicaragua und Costa Rica gemeint.

3 Obgleich im Fall von El Salvador kein Zweifel an der Rolle der USA als treibender Kraft hinter dem Rückzug der Militärs von der politischen Bühne besteht, heißt das jedoch keineswegs, daß allein äußere Faktoren die Einführung der Demokratie bewirkt haben. Im Falle Guatemalas scheinen beispielsweise externe und interne Gründe gleichermaßen wirksam gewesen zu sein. Die Interessen der US-Regierung, in El Salvador eine demokratische Regierung mit dem Ziel zu etablieren, der US-amerikanischen Unterstützung der salvadorianischen Streitkräfte einen Anschein von Legitimation zu verschaffen, zugleich der Ausstrahlung des nicaraguanischen Beispiels zu begegnen sowie mit der Ablösung der Militärs in Honduras eine bessere Legitimation für die Führung des Contra-Kriegs gegen Nicaragua zu erreichen, verband sich mit dem Interesse der guatemaltekischen Militärs, wieder Wirtschafts- und Militärhilfe aus den USA zu erhalten, wozu ebenfalls die Einführung der Demokratie angebracht war (Flora/Torres-Rivas 1989: 40). Darüber hinaus mögen weitere endogene Gründe eine Rolle gespielt haben, die Lechner als Faktoren für den Rückzug der Militärs in Südamerika nennt: Die soziale Integration durch den Markt fand in Mittelamerika kaum statt und so stellte die Demokratie den Versuch dar, eine politische Integration zu bewerkstelligen, die mit den repressiven Methoden der Militärherrschaft besonders ab Mitte der 70er Jahre nicht mehr gelang (Lechner 1993: 70; 1994: 34). Insgesamt ist aber weitgehend unstrittig, daß endogene Ursachen in Mittelamerika vergleichsweise weniger relevant waren als bei der Wiedererrichtung der südamerikanischen Demokratien. Die mittelamerikanischen Demokratien der 80er Jahre sind eher verhängt denn erstritten worden. Die Einführung der Demokratie fand dann auch - anders als in Südamerika - weitgehend unter Ausschluß der oppositionellen demokratischen Bewegungen der jeweiligen Länder statt (Torres-Rivas 1990a; 1990b; Lungo 1993; Samour 1994). 
nach »Freedom House« in die mittlere Gruppe der politischen Systeme, werden also weder den $76 »$ freien «, noch den 53 »nicht freien «, sondern der Gruppe der 62 »teilweise freien« Länder zugeordnet. Sie sind als nicht konsolidiert zu betrachten. Nur im Fall Costa Rica kann man von einer gefestigten Demokratie sprechen. Sie gründet sich auf eine längere demokratische Tradition, eine gerechtere Einkommensverteilung, einen höheren Lebensstandard und eine höhere Zustimmung in der Bevölkerung. Warum ist es bislang nicht gelungen, in den anderen Ländern Mittelamerikas die Demokratie zu konsolidieren? Wo liegen die Schwierigkeiten?

Zunächst soll bei der Verfolgung dieser Fragen auf einige wichtige Untersuchungen eingegangen werden (Kap. 2). Da diese Interpretationsversuche meines Erachtens viele wichtige Fragen gar nicht berühren, folgt die Auflistung einiger politischer Faktoren, die mir für das Studium des Themas von Bedeutung $\mathrm{zu}$ sein scheinen, aber in den vorliegenden Studien kaum vorkommen (Kap. 3). Auch zum Thema der Parteien erscheint es mir notwendig, einige ergänzende Bemerkungen zu machen und die Frage nach ihrer Repräsentativität und Integrationsfunktion zu stellen (Kap. 4). Mit der anschließenden Darstellung der geringen Akzeptanz, die die politischen Regimes einschließlich der Parteien in der Bevölkerung finden (Kap. 5), stoßen wir auf das eigentliche Problem für die Konsolidierung der demokratischen Regimes: Die Bevölkerung lehnt bloß symbolische politische Handlungsoptionen ab und artikuliert sich in anderen politischen Handlungsformen (Kap. 6). Abschließend werden nicht-symbolische Handlungsoptionen vorgestellt, die in einem komplementären Verhältnis zu den repräsentativ-parlamentarischen stehen (Kap. 7).

\section{Theoretische Annäherungen und ihre Grenzen}

Die vorliegenden theoretischen Annäherungen an Mittelamerikas neue Demokratien kreisen - grob zusammengefaßt - um drei hauptsächliche Fragerichtungen. Da ist zunächst die aus der Modernisierungstheorie bekannte makrosoziologisch orientierte Grundfrage: Welches sind die Voraussetzungen, die in einer Gesellschaft erfüllt sein müssen, damit Demokratie möglich wird? Hierbei werden die Chancen für die Entstehung und die Festigung der Demokratie in Abhängigkeit vom Entwicklungsstand des jeweiligen Landes gesehen, wobei der wirtschaftlichen Entwicklung das entscheidende Gewicht beigemessen wird. Dieser makrosoziologische Ansatz trägt deutlich deterministische Züge.

Den zweiten Komplex bildet die Untersuchung der Transformationsprozesse, d.h. der Modalitäten der institutionellen und normativen Übergänge vom autoritären zum demokratischen Regime. Die Fragerichtung versteht sich als Gegensatz zur »makrohistorischen komparativen Soziologie«, der 
auch der modernisierungstheoretische Ansatz zugerechnet wird, in dem ) Geschichte stattfindet, ohne daß irgend jemand jemals irgend etwas tut (Przeworski 1991: 96). Die neue Orientierung drückt sich im Wechsel von der sozialstrukturellen zur akteurszentrierten Perspektive aus. Ihr besonderes Interesse gilt den Veränderungen auf der staatlichen Ebene und dem hicrauf bezogenen Handeln der Akteure. Die demokratische Qualität dieser Handlungen wird als konstituierend für das Gelingen oder Scheitem der nachfolgenden Demokratie angesehen.

Aus der Transitionsforschung hat sich eine dritte Richtung herausentwikkelt, die sich den Problemen der Konsolidierung widmet (Bos 1994). Dieses Thema wurde in bezug auf Mittelamerika virulent, als sich abzeichnete, daß trotz der als geglückt angesehenen Transitionen die demokratische Konsolidierung der neuen Regimes nicht gelingen wollte. Obgleich dieser Ansatz thematisch und methodisch eng an die Transitionsforschung anknüpft, erscheint er mir einer gesonderten Darstellung wert, weil er m.E. den Punkt markiert, an dem die vorliegenden Ansätze aus konzeptionellen Gründen ihr Thema nicht mehr bewältigen können. ${ }^{4}$ Hier gelangen die Interpretationen an die Grenzen, die ihnen durch ihre Beschränkung auf das Institutionen- und Normengefüge gesetzt sind.

\subsection{Modernisierungstheoretisch inspirierte Interpretationen}

Wenn wie heutzutage weitverbreitet unter Demokratie ein bestimmtes Institutionengefüge, ein Regelsystem, kurz (wie schon seit Schumpeter): eine Methode verstanden wird, gilt sie als eine politische Organisationsform, die prinzipiell allgemeingültig und unabhängig vom jeweiligen Kontext realisierbar ist ${ }^{5}$ : Demnach wird Demokratie möglich, wenn bestimmte Voraussetzungen erreicht sind. ${ }^{6}$

In seiner klassischen Studie »Political Man« von 1959 fragt Lipset nach den Voraussetzungen für die Demokratie und kommt zu dem Ergebnis, daß

4 So Schmitter (1993: 3), Linz/Stepan (1996: 14f); zur Kritik siehe O'Donnell (1996).

5 "Die demokratische Methode ist diejenige Ordnung der Institutionen zur Erreichung politischer Entscheidungen, bei welcher einzelne die Entscheidungsbefugnis vermittels eines Konkurrenzkampfes um die Stimmen des Volkes erwerben. (...) Demokratie bedeutet lediglich, daß das Volk die Möglichkeit hat, die Männer, die es beherrschen sollen, zu akzeptieren oder abzulehnen. (...) Demokratie ist die Herrschaft des Politikers« (Schumpeter 1993: 428, 452). Dazu bemerkt Habermas: »Diese sozialtechnische Auffassung unterstellt die Demokratie als ein Modell, das sich vom realen Prozeß ihres gesellschaftlichen Ursprungs ablösen und, Anpassungen eingerechnet, auf beliebige Situationen übertragen läßt« (Habermas 1977: 10).

6 Dieses institutionen- und normenzentrierte Demokratieverständnis benennt als Bezugsrahmen explizit die Jiberale Demokratie und schließt umfassendere oder überhaupt andere Demokratievorstellungen ausdrücklich aus, darunter auch solche, die neben den individuellen kollektive Rechte, besonders wirtschaftliche und soziale, stärker in den Vordergrund stellen (beispielhaft: Diamond, Linz, Lipset 1990: 3). 
diese hauptsächlich in der wirtschaftlichen Entwicklung zu sehen seien. Er hatte diese anhand von vier Indikatoren untersucht: Reichtum, Industrialisierung, Verstädterung und Erziehung. Damals stellte er fest, daß in den demokratischen Ländern diese vier Faktoren sämtlich höher entwickelt waren als in den nicht-demokratischen (Lipset 1993: 45). Er kehrte dann die Interpretation seiner Ergebnisse um und definierte so die Entwicklung dieser vier Faktoren als Bedingung für die Demokratie. Noch 35 Jahre später findet er seine Ergebnisse von 1959 durch neue Entwicklungen bestätigt (Lipset et al. 1994: 49). Als wichtigster Maßstab für Demokratie gilt in der neuen Untersuchung weiterhin das Pro-Kopf-Einkommen (ebd.: 13). Ähnlich dem Vorgehen in der Studie von 1959 schließt Lipset aus der Feststellung, derzufolge die entwickelten Länder demokratischer seien, daß Länder mit geringer kapitalistischer Entwicklung kaum Chancen auf demokratische Entwicklung aufweisen (ebd.: 16). Auf Mittelamerika bezogen wäre daraus zu folgem, daß die Chancen auf eine gefestigte Demokratie angesichts der gegebenen wirtschaftlichen Entwicklung schlecht stünden.

\section{2 Übergänge zur Demokratie}

Vergleichbare Korrelationen von wirtschaftlicher Entwicklung und Demokratie werden häufig aufgestellt - z.B. Diamond/Linz/Lipset (1990: 9ff) oder Diamond (1992: 106) - und manche Autoren halten derartige Postulate gar für inzwischen durchgesetzt. ${ }^{7}$ Wenngleich auch die empirische Feststellung nicht zu bestreiten ist, daß wirtschaftlich entwickelte Länder häufiger über demokratische Regimes verfügen als gering entwickelte und auch der Umkehrschluß belegbar ist, so äußern andere Autoren Zweifel, ob auf der allgemeinen Ebene von Voraussetzungen überhaupt Aussagen über Demokratie zu machen sind.

Mit der Betrachtung der Transitionsprozesse wendet sich das Interesse den Einzelheiten der historischen Umbrüche zu. Wie in den modernisierungs-

7 So schreibt Johannes Berger in dem der Modernisierungstheorie gewidmeten Heft des "Leviathan《 zur sogenannten Lipset-These (»Democracy is related to the state of economic development. The more well-to-do a nation, the greater the chances that it will sustain democracy《): »Wenn ich recht sehe, darf diese Annahme heute im wesentlichen als bestätigt gelten« (Berger 1996a: 11; 1996b: 57). Tatsächlich jedoch kann von einer einhelligen Auffassung über die Art des Zusammenhangs von wirtschaftlicher Entwicklung und Demokratie keine Rede sein, im Gegenteil. Immer öfter werden allgemeine Aussagen über eine solche Beziehung für untauglich gehalten. Die Äußerungen zum Thema werden differenzierter (Karl 1990; Karl/Schmitter 1991) oder bestreiten gar jeglichen Zusammenhang. So fragt Tetzlaff: »Ist Demokratie eine Folge oder eine Voraussetzung für wirtschaftliches Wachstum und gesamtgesellschaftliche Entwicklung? Die Antwort der vergleichenden Sozialforschung lautet: Es gibt keine eindeutige Korrelation zwischen den beiden GröBen« (Tetzlaff 1992: 12) und Beyme stellt fest: »Inzwischen ist die Einsicht gewachsen, daß es keine fixierbaren Prärequisiten der Demokratie gibt« (Beyme 1994: 153). 
theoretischen Ansätzen ist auch hier ein komparatistischer Ansatz dominant, der um vorwiegend institutionenbezogene generalisierungsfähige Indikatoren bemüht ist. Anders aber als in der Modernisierungstheorie erscheinen hier die politischen Akteure als die relevanten Faktoren für die Chance der Errichtung demokratischer Regimes. Der Perspektivwechsel von der Diskussion allgemeiner Vorbedingungen hin zum Blick auf den konkreten Ablauf des Umbaus autoritärer Regimes und auf die Akteure stellt einen Fortschritt in der Analyse der konstitutiven Faktoren der Demokratie dar (Schmitter 1993: 3f; Bos 1994: 105), wie ebenso die Annahme, daß den Spezifika des jeweiligen Übergangs eine prägende Bedeutung für die Gestaltung der Demokratie zukommt.

Aber die stark staatskonzentrierte Perspektive, mit der der Institutionenund Wertewandel im Verlauf des demokratischen Aufbaus betrachtet wird $^{8}$, beeinflußt das Bild vom politischen Akteur. Als ein solcher gilt nur, wer am institutionellen und normativen Wandel teilnimmt, also in der Regel am staatlichen Handeln beteiligt ist (Bos 1994). Der Blick auf die institutionell handelnden Eliten nimmt den politischen Akteur nur zur Hälfte wahr. Die übersehene andere Hälfte besteht in den nicht-institutionellen Kräften. ${ }^{9}$ Wichtige - etwa kollektive - Akteure von prägender Bedeutung für die Demokratie befinden sich außerhalb des staatlich induzierten und untersuchten Transitionsbereichs. Die Beschränkung auf die staatliche Sphäre erschwert die Wahrnehmung solcher Kräfte, die für die Konsolidierung von großer Bedeutung sein können.

\subsection{Probleme der Konsolidierung}

Aus der Untersuchung der Transitionsprozesse lassen sich offenbar keine hinreichenden Kriterien für die Chancen einer demokratischen Konsolidierung entwickeln. Die ungeklärten Fragen lauten: Wo liegen die gesellschaftlichen Hindernisse für die Konsolidierung? Und: Verfügen die Länder Mittelamerikas überhaupt über die Möglichkeiten, ihre demokratischen

8 Einer der wichtigsten Transitionsforscher, Przeworski, merkt selbstkritisch an, aufgnund der Fixierung auf den Staat den Zusammenhang zwischen den nunmehr umgebauten politischen Systemen und der Demokratie nicht gesehen zu haben. $\gg$ Beim zweiten Thema (neoliberale Staatszerstörung, KDT) haben wir über die Demokratisierungsprozesse gesprochen, ohne das Verhältnis von Staat und Demokratie zu erkennen. Ich erinnere mich, als Guillermo (O'Donnell, KDT) über dieses Problem zu sprechen begann, erschrak ich und dachte: wir haben 15 Jahre über nichts anderes als den Staat geredet und hinter dem Wort 'Staat' ist er verschwunden. Immerhin haben wir 15 Jahre nur über die Transitionen und die Demokratie gesprochen. Wenn wir dieses Verhältnis untersuchen, so kommen wir heute, wie ich glaube, zu folgendem Schluß: Es gibt keine Demokratie ohne Staat« (Alarcón, O’Donnell, Przeworski 1994: 105).

9 Die Transitionsvereinbarungen in Nicaragua wurden beispielsweise erheblich von den großen Streikbewegungen beeinflußt, die auf die Wahlniederlage der Sandinisten folgten (Ríos 1995). 
Regimes zu konsolidieren und worin bestehen diese? Welche sozialen Akteure kommen in Frage?

Seit Beginn der 90er Jahre rücken diese Fragen in den Vordergrund und damit auch die Untersuchung der gesellschaftlichen Konstellationen in den jeweiligen Ländern. Vor allem die nicht-mittelamerikanische Diskussion bleibt dabei weiterhin der definitorischen Anstrengung um allgemeingültige Kriterien verhaftet, die für die demokratische Konsolidierung Voraussetzung seien. ${ }^{10}$ Thematisiert werden vor allem die einer Konsolidierung hinderlichen Elemente wie institutionelle Mängel, autoritäre Relikte, überkommene Strukturen. Demgegenüber bleiben die einer demokratischen Konsolidierung förderlichen Faktoren - gesellschaftliche Akteure, Traditionen, kulturelle Gegebenheiten, an denen angeknüpft werden könnte bemerkenswerterweise bis zum heutigen Tag jenseits der Betrachtung. Das mag am liberaldemokratischen Demokratieverständnis mit seiner Institutionenorientiertheit liegen, von dem die meisten der nicht aus der Region stammenden Untersuchungen durchdrungen sind (Bos 1994: 101). Die liberale Demokratieforschung tut sich offensichtlich schwer mit der Anerkennung der Bedeutung nicht-institutioneller Faktoren für die Konsolidierung demokratischer Regimes, denn sie kann sie nur funktional in bezug auf die Funktionsfähigkeit der institutionellen Instrumente der Demokratie würdigen.

\section{Ausgangspunkte für die Demokratisierungsdiskussion}

Die bisher referierten Ergebnisse zeigen eine auffällige Diskrepanz zu den Beiträgen aus Mittelamerika selbst, die eine intensivere Beschäftigung mit den jeweiligen gesellschaftlichen Konstellationen in den einzelnen Ländern aufweisen. Allerdings beziehen sich die wichtigsten dieser Beiträge ihrerseits kaum auf die allgemeine demokratietheoretische Diskussion außerhalb Mittelamerikas. Angesichts des kommunikativen Grabens zwischen

10 Schmitter (1993: 3), Linz/Stepan (1996: 14f), O’Donnell (1996). Letzterer legt beispielsweise die Dahlsche Polyarchie-Definition zugrunde, in der sieben Kriterien angeführt werden (Dahl 1992: 268): »1. Wahl des politischen Personals, 2. freie und gerechte Wahlen, 3. umfassendes aktives Wahlrecht, 4. passives Wahlrecht, 5. Redefreiheit, 6. freie Informationsmöglichkeit, 7. Versammlungsfreiheit.« O'Donnell ergänzt diese Kriterien noch um einige weitere (O'Donnell 1996: 35). Linz und Stepan dagegen formulieren als Kriterien für Konsolidierung zunächst ein funktionierendes Institutionengefüge und darüber hinaus: »1. freie und lebendige zivile Gesellschaft, 2. relativ unabhängige politische Gesellschaft, 3. Rechtsstaatlichkeit, 4. der demokratischen Regierung dienende staatliche Bürokratie und 5. institutionalisierte ökonomische Sphäre« (Linz/Stepan 1996: 17). Die meisten dieser Faktoren erwähnt auch Karl, ergänzt sie um das Kriterium eines zivilen Befehls über die Streitkräfte, und nimmt darüber hinaus die Frage nach den gesellschaftlichen Hemmnissen der Konsolidierung mit auf (Karl 1990: 2). Eine Untersuchung des gesellschaftlichen Demokratiepotentials in den jeweiligen Ländern und ihre Einbeziehung in die demokratietheoretische Diskussion scheint bislang nicht angestellt worden zu sein. 
der mittelamerikanischen und der außerregionalen Diskussion erscheint es mir sinnvoll, einige wesentliche Gesichtspunkte der mittelamerikanischen Forschung zu erwähnen.

Ich will im folgenden auf fünf meines Erachtens in der allgemeinen Debatte zu Unrecht übergangene Aspekte aufmerksam machen, wobei der wichtigste Punkt die Frage betrifft, ob der Staatsbürger, auf den sich die Demokratie gründen soll, überhaupt existiert. Diese Punkte werden in der mittelamerikanischen Diskussion hervorgehoben und ihre Vernachlässigung bleibt nicht ohne Folgen für die Interpretation der Demokratisierungen.

Über diese fünf Aspekte der Demokratiefrage in Mittelamerika hinaus sind noch einige weitere zu nennen, die hier aber nicht ausgeführt werden können, ohne daß ihnen deshalb geringeres Gewicht zugemessen würde. Einer davon ist der Verlust der nationalen Souveränität der Staaten Mittelamerikas, der vor allem auf wirtschaftspolitischem Gebiet stattgefunden hat. Für die Stabilisierung - oder Schwächung - eines politischen Regimes ist die Wirtschaftspolitik aber von entscheidender Bedeutung. Einen anderen Aspekt bilden die kulturellen, ethnischen und organisatorischen Traditionen der jeweiligen Bevölkerungen. Am eindrücklichsten springt die Frage der Anerkennung autochthoner Formen in Guatemala ins Auge. ${ }^{11}$

\subsection{Zwei Demokratisierungen}

Den Schlüssel für das Verständnis der Demokratisierung in Mittelamerika, die in den 80 er Jahren begann, bildet die Feststellung, daß dieser Prozeß keineswegs kontinuierlich verlaufen ist, sondern tiefe Brüche aufweist, so daß von zwei Demokratisierungen gesprochen werden kann, eine in den 80 er und eine in den 90er Jahren, namentlich im Hinblick auf zwei Länder, El Salvador und Guatemala. Bei der ersten kamen in diesen beiden Ländern die Unterschichten als politische Akteure nicht vor, sie drückte keinen gesellschaftlichen Kompromiß aus und hatte nichts mit der Vorstellung gemein, hier stritten gesellschaftliche Akteure um die Einführung und Vertiefung der Demokratie. Tatsächlich kam die Demokratisierung des politischen Systems von oben, und zwar in Folge erheblichen US-amerikanischen Drucks.

Während der nahezu synchronen Einführung der Demokratie in Honduras, El Salvador und Guatemala waren diese Länder mehr oder weniger im Kriegszustand ${ }^{12}$ und die Transitionen erfolgten unter Kontrolle der Streit-

11 Dort sind 1996 erstmals die politischen Organisationen der indigenen Völker massiv in die staatlichen Institutionen eingezogen und haben nach den Kommunalwahlen 90 der 330 Munizipien übernommen. Zur jüngsten organisatorischen Entwicklung der Mayas siehe Bastos/Camus (1995).

12 El Salvador befand sich mitten im Bürgerkrieg, in Guatemala waren zu diesem Zeitpunkt die bereits 20 Jahre andauemden bürgerkriegsähnlichen Auseinandersetzungen in einen 
kräfte. Entsprechend begrenzt war die Reichweite der Demokratisierung. Sie beschränkte sich auf wenige institutionelle und einige normative Bestandteile eines demokratischen Staatswesens (weitgehend unverändert blieben das Justizwesen, der beherrschende Einfluß der Streitkräfte, der Polizeien etc.) sowie auf die Neustrukturierung und selektive Zulassung politischer Akteure zum demokratischen System, wovon der Großteil der Opposition ausgeschlossen blieb. ${ }^{13}$ Die Transitionen der 80er Jahre waren nicht vornehmlich auf die Demokratisierung der Gesellschaft gerichtet und hatten nicht die Stärkung, sondern die Schwächung der gesellschaftlichen Träger eines demokratischen Prozesses zum Ziel. Die Installierung der Demokratie war als eine Art Aufstandsprävention gegen die jeweiligen Gesellschaften gerichtet, wie am deutlichsten das salvadorianische Beispiel der frühen 80er Jahre zeigt (Bonner 1984: 181ff). Die Bemühungen der Opposition um eine Vertiefung des Demokratisierungsprozesses sind von den autoritären Demokratien unterbunden worden. In El Salvador und Guatemala hat diese Art von autoritär implementierter Demokratie nicht zur politischen Integration, sondern zur Polarisierung, nicht zur Beendigung, sondern eher zur Verlängerung des Krieges beigetragen.

In El Salvador hat der zwölf Jahre währende Bürgerkrieg schließlich das autoritäre Modell der 80er Jahre zum Einsturz gebracht und ein neues auf die Tagesordnung gesetzt. So hat keine graduelle Erweiterung der Demokratie, sondern erst der Bruch mit dem autoritären demokratischen System die Möglichkeit für einen Neubeginn freigesetzt. Über die kathartische Wirkung eines solchen tiefen Einschnitts für einen demokratischen Neubeginn bemerkt Merkl:

»Um einen starken demokratischen Mythos zu erzeugen, der die Entwicklung der Demokratie in der Zukunft beflügelt, ist wahrscheinlich viel eher ein schreckliches Ereignis erforderlich wie ein Krieg, eine ausländische Intervention oder eine gewaltsame Revolution mit anschlieBendem Zusammenbruch eines autoritären Systems als ein gradueller Übergang« (Merkl 1994: 83-84).

Das Ende der autoritären und das Fundament einer neuen Demokratie in El Salvador ist mit dem Friedensschluß zwischen Regierung und FMLN im Januar 1992 besiegelt worden und möglicherweise wird der Friedensschluß in Guatemala 1996 eine ähnliche Funktion haben. Erst vom Zeitpunkt der Verhandlungen über das Friedensabkommen an begann die Opposition in

Ausroitungsfeldzug durch die Regierung übergegangen und in Honduras ging der Umbaus des Landes in einen US-Militärstützpunkt und in ein Aufmarschgebiet der antisandinistischen Contra vonstatten.

13 Bendel/Krennerich 1996. Auf den Ausschluß der Opposition bei den Übergängen der 80er Jahre weisen fast alle mittelamerikanischen Autoren hin, siehe etwa Torres-Rivas 1990a, 1990b; Lungo 1993; Samour 1994. In der theoretischen Debatte wurden die Demokratien der $80 \mathrm{er}$ Jahre als beschränkte Demokratien, Demoknatien von geringer Intensität (Torres-Rivas) oder als Fassadendemokratien, (Solórzano) bezeichnet. 
beiden Ländern einen nennenswerten Einfluß zu erlangen. Der Guerilla wurde - und von dieser vermittelt auch der zivilen Opposition - eine gewisse Mitgestaltungskompetenz zugebilligt.

Damit ist nicht gesagt, daß es den bis dahin oppositionellen gesellschaftlichen Kräften bisher gelungen wäre, an den politischen Entscheidungen in umfangreichem Maß teilzunehmen. Zwar sind nach 1992 in El Salvador in Honduras schon in den 80er Jahren - zwei für die Demokratie konstitutive Elemente eingeführt worden, ein öffentlicher Raum, in dem der repressions- und angstfreie politische Diskurs möglich wird, und - langsam - die Schaffung einer Rechtsstaatlichkeit, die diesen Raum zu schützen vermag. Doch ist hervorzuheben, daß diese Elemente unter Bedingungen der Ausnahmesituation militärischer Befriedungen unter Aufsicht der Vereinten Nationen erstritten wurden, die Ausdruck eines temporär veränderten politischen Kräfteverhältnisses waren. ${ }^{14}$ Wieweit durch die mittlerweile eingetretene Normalisierung der Bedingungen auch wieder traditionelle Kräfteverhältnisse in den Vordergrund treten, bleibt abzuwarten. Aufgrund der Bedingungen ihrer Einführung sind Rechtsstaatlichkeit, öffentlicher Raum und andere demokratische Fortschritte keineswegs so unangefochten, wie sie scheinen mögen. Nicht nur in El Salvador, sondern auch in Guatemala und in Honduras wirkt sich die Tatsache, daß auch die Errichtung der zweiten Demokratie nicht auf den Triumph einer gesellschaftlichen Bewegung gegründet ist, negativ auf den demokratischen Gehalt und die Stabilität der Institutionen und Normen aus.

\subsection{Reichweite der zweiten Demokratisierungen}

Die Demokratien Mittelamerikas - mit Ausnahme Costa Ricas - zeichnen sich durch ihre geringe gesellschaftliche Tiefe aus. Die demokratischen Veränderungen beschränken sich auf die staatliche Sphäre im engeren Sinne und haben auf der Ebene der Institutionen (saubere Wahlen, Parteienkonkurrenz, Regierungswechsel, Justizreform usw.) und auf der der Normen (reale Gewaltenteilung, Menschenrechte, Meinungs- und Versammlungsfreiheit usw.) stattgefunden. Andere gesellschaftliche Bereiche wurden dagegen kaum oder nicht erfaßt. Mechanismen der Konsultation oder gar Möglichkeiten der Partizipation am politischen Entscheidungsprozeß gibt es für nicht-parlamentarische Organisationen bislang nicht. Demgemäß fehlen institutionelle Wege zur Lösung sozialer Konflikte, was eine

14 In El Salvador war das Instrument der zivilen Einflußnahme der »Foro de Concertación Económica y Social«, der in den Friedensverhandlungen auf Druck der FMLN eingerichtet worden war; in Guatemala die »Asamblea de la Sociedad Civil und in Nicaragua geschah dies im Rahmen der Verhandlungen um die Übergabe der Regierungsgewalt von den Sandinisten an die konservative Präsidentin Chamorro. Alle drei Versuche zielten auf eine Einbeziehung der Zivilgesellschaft in die Verhandlungen über den Aufbau des neuen Regimes. 
häufig gewalttätige Austragung der Konflikte nach sich zieht und die Gesellschaften enorm polarisiert. Im Mangel an Vermittlungsmechanismen von Gesellschaft und Staat setzt sich die überkommene Abdrängung der Gesellschaft von der politischen Sphäre fort. Die integrative Kraft des demokratischen Institutionen- und Normengefüges ist entsprechend gering. Das einzige Instrument zur Herstellung der politischen Integration bleiben die Wahlen.

\subsection{Neuerrichtung statt Wiedererrichtung der Demokratie}

In den meisten Studien über lateinamerikanische Transitionen werden südamerikanische Erfahrungen mit der Wiedererrichtung der Demokratie reflektiert. Dementsprechend bezeichnet Konsolidierung dort einen Prozeß, der sich weitgehend auf eine bestehende gesellschaftliche Akzeptanz des demokratischen Institutionen- und Regelwerks stützen kann. In Mittelamerika bezeichnet Konsolidierung dagegen einen mühseligeren Prozeß. Es geht hier nicht um die Wieder-, sondern die Neuerrichtung der Demokratie. Der Rückgriff auf eine - durch die autoritären Regimes nur unterbrochene - historische Akzeptanz des demokratischen Regimes ist hier ausgeschlossen. Wird das übersehen, werden die Schwierigkeiten für die Konsolidierung der Demokratie leicht unterschätzt.

\subsection{Die politischen Regimes}

Nach den von äußeren Interessen induzierten Regimewechseln war besonders außerhalb Mittelamerikas die Neigung verbreitet, eine effektive Repräsentativität schon dann als gegeben zu unterstellen, als die ersten sauberen Wahlen abgehalten worden waren, also ab Anfang der 80er Jahre. Jedoch hat das repräsentative Prinzip in den meisten Ländern Mittelamerikas kaum eine Tradition. Wenn in Darstellungen diesem eine Funktionsfähigkeit in Analogie zu den entwickelten Demokratien unterstellt wird, ist eines der zentralen Probleme bereits ausgeklammert: die mangelnde Repräsentativität der Parteien und des politischen Systems insgesamt. Angesichts der langen autoritären Vorgeschichte ist hier die Frage relevant, ob die gewählten Kandidaten tatsächlich den Wählerwillen repräsentieren, also repräsentativ sind. In einigen Ländern etwa ist gerade diese Gleichsetzung völlig unberechtigt, so in Guatemala und bis 1994 auch in El Salvador.

Auch die Annahme, die Parlamente fungierten als Ort der über die Parteien vermittelten Einflußnahme gesellschaftlicher Interessen auf die Politik der Exekutive, führt in die Irre. Wie in den meisten lateinamerikanischen Ländern erfüllen auch in Mittelamerika die Parlamente diese Funktion nur unzureichend. Es handelt sich um zentralistische Präsidialdemokratien, in denen der vom Volk direkt gewählte Präsident mit der Autorität ausgestattet 
ist, Politik weitgehend nach eigenem Ermessen zu realisieren, während die Parlamente eine geringe Rolle spielen. Anders als in parlamentarischen Demokratien und in einigen präsidialen Systemen, wie den USA, wird die Exekutive von der Legislative nicht oder kaum kontrolliert. Die Stellung des Präsidenten ist über die anderer Staatsorgane hinausgehoben. Autoritäre Regierungspraktiken werden der Exekutive geradezu in den Schoß gelegt. ${ }^{15}$ Die Geringschätzung des von den Parlamentsparteien repräsentierten Wählerwillens ist somit Wesensbestandteil dieser politischen Systeme. Die Parteien haben auf die von ihnen an die Macht getragene Exekutive noch nicht einmal Einfluß, was in diesem Fall auch für die sonstige Ausnahme Costa Rica zutrifft (für Costa Rica siehe Rojas 1994; allgemein: O'Donnell 1994: 18ff). Die in der Regel impliziten Unterstellungen in bezug auf Repräsentativität und Parlamentarismus weisen den Institutionen Partei und Parlament Funktionen zu, die diese nicht ausfüllen können.

\subsection{Demokratien ohne demokratischen Souverän}

Die schwache Präsenz des Staatsbürgers (citoyen, ciudadano)

Die größte Beschränkung für die demokratische Konsolidierung liegt im Fehlen eines demokratischen Souveräns. Die Bevölkerung ist im Besitz der formalen staatsbürgerlichen Attribute, doch verfügt ein Großteil von ihr über keine politische Artikulationsfähigkeit.

Obgleich in modemen Demokratien in bezug auf politische Einflußnahme nicht vom einzelnen Bürger, sondern von Verbänden als den politischen Subjekten ausgegangen wird, muß dennoch das einzelne Individuum, auch wenn es nur in organisierter Form politisch einflußfähig werden kann, über diejenigen Eigenschaften verfügen, die es als Bürger überhaupt erst konstituieren, um seine Interessen vernünftig in kollektive Formen umsetzen zu können. Diese Eigenschaften bestehen nicht nur aus dem formalen Rechtsstatus, sondern auch aus der individuellen Kompetenz zur politischen Artikulation sowie angesichts der gegebenen Verbandsorientiertheit der Demokratien auch aus einer kollektiven Handlungsmöglichkeit.

Die Ausübung des Bürgerstatus als Fundament für eine funktionierende Demokratie hat dreierlei zur Voraussetzung, a. den Rechtsstaat, b. eine öffentliche Sphäre und c. materielle Mindestbedingungen, die auch kulturelle und informative Möglichkeiten einschließen. Es ist vor allem diese letztere Bedingung, die für die Bevölkerungsmehrheit in Mittelamerika nicht erfüllt ist. Es handelt sich um Gesellschaften, in denen ein erheblicher Bevölkerungsanteil strukturell aus den Marktbeziehungen ausgeschlossen ist, in denen mehr als zwei Drittel der Bevölkerung in Armut und die Hälfte

15 Am Beispiel Guatemalas bezeichnet Gálvez das Präsidialregime als »Quelle der Unregierbarkeit« (Gálvez 1995: 32). 
gar in extremer Armut leben, in denen hohe Arbeitslosigkeit und noch höhere Unterbeschäftigung herrschen, in denen öffentliche Dienstleistungen wie Gesundheit und Erziehung für die arme Bevölkerung kaum noch zur Verfügung stehen. ${ }^{16}$ Die bloße Gewährung des Status eines bürgerlichen Rechtssubjekts macht die Individuen noch lange nicht zu Bürgern im praktischen Sinne ${ }^{17}$, weil es ihnen nicht nur an

》Gütern und materiellen Dingen fehlt, sondern weil sie darüber hinaus insofern politisch machtlos sind, als sie nicht über die psychologisch-kulturellen Bedingungen verfügen, um eine Selbstwahrnehmung ihrer gesellschaftlichen Lage zu entwickeln und dementsprechend die organisierte politische Verteidigung ihrer eigenen Interessen zu betreiben«, stellt Torres-Rivas fest und fragt weiter: »Welche staatsbürgerlichen Eigenschaften können unter derartigen materiellen und kulturellen Gegebenheiten entstehen oder gefestigt werden, welche politische Demokratie kann man damit aufoauen? « (Torres-Rivas 1992: 138).

Diese mittelamerikanischen Bürger, die als Staatsbürger nur formal existieren, stellen in jedem Land, mit Ausnahme Costa Ricas, bei weitem die Bevölkerungsmehrheit. ${ }^{18}$ Die Mehrheit in Mittelamerika, die den Unterschichten angehören, sind zwar Staatsbürger, aber sie sind im Rahmen der

16 Der unter der Armutsgrenze lebende Bevölkerungsanteil lag im mittelamerikanischen Durchschnitt 1985 bei $71,7 \%$. Er betrug in Guatemala $82,6 \%$; in El Salvador $86,9 \%$; in Honduras 78,8\%; in Nicaragua $68,7 \%$; in Costa Rica $28,1 \%$ und in Panamá $40,0 \%$ (FLACSO 1995: 104). Der Schwerpunkt der Armut liegt traditionell in ländlichen Gebieten. 1985 lebten auf dem Land $83 \%$ der Bevölkerung in Armut, in städtischen Gebieten $58 \%$. In den $80 \mathrm{er}$ Jahren hat eine starke Pauperisierung in der Region stattgefunden. Die Wachstumsrate der Armut lag doppelt so hoch wie die der Bevölkerung. Diese neue Armutswelle ist ein vornehmlich städtisches Phänomen. Die Einkommensverteilung ist in Mittelamerika - außer Costa Rica - sehr ungleich: 1980 verfügte das ärmste Bevölkerungsfünftel über knapp 4\% des regionalen Einkommens, während das reichste Fünftel 57\% vereinnahmte (Menjivar/ Trejos 1992: 10, 34, 70, 74). In den folgenden Jahren ist der Anteil des untersten Fünftel weiter zurückgegangen: In El Salvador (1990-91) auf $3,5 \%$; in Guatemala (1989) auf 2,1\%, in Honduras (1989) auf 2,7\% und lag in Nicaragua (1993) bei 4,2\% (Vilas 1996: 471).

17 Ein erwerbs- und damit einkommensloser oder im informellen Sektor sein Überleben suchender Bürger verfügt zweifellos über sehr eingeschränkte Möglichkeiten, sich zu informieren, ein politisches Interesse zu entwickeln und sich schließlich am organisierten politischen Meinungsaustausch zu beteiligen. Da seine über familiäre Beziehungen hinausgehende soziale Integration abnimmt, hat er immer weniger die Chance, sich an kollektivem politischen Handeln zu beteiligen. Folgenreich ist daneben, daß die Menschen ohne Erwerbsarbeit, Unterbeschäftigte, die große Zahl der im sogenannten »informellen Sektor« Aktiven und Kleinstuntemehmer (microempresarios) von politischen oder gewerkschaftlichen Organisationen kaum noch erreicht werden und infolgedessen ihrer kollektiven politischen Handlungsoptionen verlustig gehen.

18 Zahlreiche Untersuchungen in westlichen Industrieländern haben ergeben, daß (liberal) demokratische Werte in den unteren Bevölkerungsklassen und dort besonders unter der unpolitischen Mehrheit - weniger verbreitet sind als in den gebildeteren Schichten und dort vor allem bei den politisch interessierten Eliten. Die Unvollkommenheit des Bürgerstatus in den Unterschichten dient in konservativen politischen Theorien als Beleg für das Postulat, daß die Funktionsfähigkeit der Demokratie wesentlich von den Eliten abhängt und durch erweiterte Bürgerbeteiligung eher gefährdet als gestärkt wird. Dementsprechend wird die Teilnahme an Wahlen als ausreichende Beteiligung am politischen Entscheidungsprozeß angesehen. 
gegebenen Demokratien politisch handlungsunfähige Staatsbürger. Die mittelamerikanischen Demokratien sind weitgehend Demokratien ohne Staatsbürger. ${ }^{19}$ Daher besteht wenig Grund zur Hoffnung auf eine Konsolidierung der Demokratie, wahrscheinlicher ist das Gegenteil.

\section{Die Parteien}

Vertretung und Vermittlung gesellschaftlicher Interessen in der staatlichen Sphäre ist in Mittelamerika exklusiv den Parteien vorbehalten. Die Parteien tragen auf diese Weise zur politischen Integration bei - doch aufgrund dieses institutionellen Kanalisierungsmonopols gesellschaftlicher Interessen behindern sie sie zugleich. Eine Repräsentation nicht-parteiförmig organisierter kollektiver Interessen ist innerhalb des institutionellen Systems der Demokratien nicht vorgesehen, sondern wird in den vorpolitischen Raum abgedrängt, in der Regel auf die Straße. Nicht-parteiförmige Organe der Zivilgesellschaft bleiben von der politischen Mitgestaltung ausgeschlossen. Die Repräsentativität der Parteien ist in den meisten Fällen gering, auch handelt es sich nicht um Mitgliederparteien. Ihre Funktion ist eher eine von 'Wahlstimmen-Sammelstellen' (Offe 1980: 32). Nur in wenigen Ausnahmen sind die Parteien Mittelamerikas politischer Ausdruck breiter gesellschaftlicher Strömungen, in der Mehrheit handelt es sich um Klientelparteien (Rojas 1995: 122). Zum Fortbestand dieses Zustands haben die langen Verbote oppositioneller Parteien unter den Militärregimes beigetragen, mit denen das repräsentierte politische Spektrum stets eng begrenzt gehalten wurde. In den wenigen Fällen der Zulassung oppositioneller Parteien taten - besonders im El Salvador der 70er Jahre - die Wahlfälschungen ein übriges, um die Distanz der Bürger zum Parteiensystem insgesamt zu fördem. Oppositionelle Interessen wurden so immer mehr im illegalen oder halblegalen Raum, aber nicht durch Parteien, organisiert. Das wirkt heute noch in schwachen Parteistrukturen nach. Das Parteiensystem kann die ihm zugedachte Rolle der politischen Repräsentation verschiedener gesellschaftlicher Sektoren gegenüber dem Staat nur unzulänglich erfüllen (vgl. Maihold 1994: 212ff).

Die Krise der mittelamerikanischen Parteien hat zum anderen mit ihrer schwachen Stellung im Präsidialsystem zu tun. Die Parlamentsparteien sind deswegen in der Mitgestaltung der Regierungspolitik nicht sehr aktiv und spielen bei der Lösung nationaler Probleme kaum eine Rolle. Viele von ihnen konzentrieren statt dessen Interesse und Aktivitäten stärker auf den Staatsapparat und seine Ressourcen mit dem Ziel der Befriedigung ihrer Partikularinteressen (Torres-Rivas 1990b: 58ff; O’Donnell 1994: 20).

19 O'Donnell spricht von »Demokratien mit Staatsbürgerschaften von geringer Intensität« (O'Donnell 1993: 75ff). 
Weit verbreitete Phänomene wie Korruption und Nepotismus durch die Parteien innerhalb der staatlichen Institutionen haben in ihrer Funktionsbeschneidung in Präsidialdemokratien eine ihrer Wurzeln.

Torres-Rivas macht für die 'Unregierbarkeit' einiger Gesellschaften, etwa der Nicaraguas, einen 'Parteienexzeß' dieser klientelistischen Notabelnparteien verantwortlich, in dem diese sich in $\gg$ parteipolitischem Kannibalismus und gegenseitiger Selbstzerfleischung « ergehen (Torres-Rivas 1994: 63). ${ }^{20}$ Selbst in der stabilsten Demokratie Mittelamerikas, in Costa Rica, haben die Parteien Schwierigkeiten, eine politische Kontinuität über die Wahlperioden hinaus aufrechtzuerhalten (Rojas 1995: 123).

Aus der mittelamerikanischen Parteienlandschaft heben sich jedoch zwei Parteien ab. Die beiden aus der Guerilla hervorgegangenen Linksparteien FSLN (Nicaragua) und FMLN (El Salvador) sind Mitgliederparteien ${ }^{21}$, deren Anhang vorwiegend den verarmten unteren Bevölkerungsschichten entstammt. Beide Parteien, die zugleich über bestimmenden Einfluß in den größten Gewerkschaften in ihren Ländern verfügen, haben die Vertretung der Interessen dieser Schichten auf ihre Fahnen geschrieben.

FSLN und FMLN erklären, eine möglichst breite Einbeziehung der Bevölkerung in die politischen Entscheidungen anzustreben. Grundsätzlich soll die Beteiligung der Bürger nicht auf die Parteien beschränkt bleiben, sondern weitere gesellschaftliche Organisationen umfassen. Sie soll über den Rahmen der liberalen Parteiendemokratie hinausgehen und auf die Aufhebung der Trennung zwischen Gesellschaft und politischer Sphäre zielen. ${ }^{22}$

20 Beispiel Nicaragua: Zu den Präsidentschafts-, Parlaments- und Kommunalwahlen 1996 traten 33 Parteien an. Um das Präsidentenamt bewarben sich 23 Kandidaten. Nahezu jede politische Strömung trat in mehrere Parteien aufgespalten und diese wiederum mit eigenem Präsidentschaftskandidaten an. Die Gründe für diese Zersplitterung wiederholen sich in ganz Mittelamerika: Sie liegen erstens im Partikularinteresse des jeweiligen Wirtschaftsclan- bzw. Sippenchefs, staatliche Pfründe für die eigene Klientel zu nutzen; in Verfolgung dieses Ziels sind Parteispaltungen nichts Unübliches. Zweitens liegen sie im Mangel an Bereitschaft zu Kooperation und Unterordnung vor allem in der Eroberung der Spitzenposition, der deutlich machistische Züge trägt. Nicaraguas Konservative haben sich allein aus diesem Grund in den letzten Jahren mehrfach gespalten. Ebenso ist die Bildung einer politischen Mitte aus mehreren Parteien an ebendiesem Verhalten gescheitert.

21 Die FSLN hat 336.000 Mitglieder, wie die Einschreibungskampagne im Frühjahr 1995 ergab. Damit ist sie eine der größten Parteien Lateinamerikas (Nicaragua hat eine Bevölkerung von knapp viereinhalb Millionen). Die FMLN ist erheblich kleiner.

$22 »$ Demokratie heißt nicht nur Wahlen. Es ist sehr viel mehr. Für einen Revolutionär, einen Sandinisten bedeutet es Beteiligung des Volkes an den politischen, wirtschaftlichen, sozialen und und kulturellen Angelegenheiten. Je mehr die Bevölkerung daran teilnimmt, um so demokratischer sind diese Angelegenheiten. Die Demokratie beginnt im wirtschaftlichen Bereich, wenn die sozialen Ungleichheiten aufhören zu bestehen, wenn die Arbeiter und Bauern ihr Lebensniveau verbessern. Dann entsteht die wirkliche Demokratie, vorher nicht. Sind diese Ziele erst einmal erreicht, dehnt sie sich sofort auf andere Be- 
In der Praxis ist dieses Modell schon bald gescheitert. Die FSLN ist nach den Wahlen 1984 zum parlamentarisch-repräsentativen Modell übergegangen und auch die FMLN hat sich im Zuge ihrer Integration in das politische System El Salvadors ab 1992 umorientiert.

Dennoch haben beide Parteien programmatisch an der Vertretung der Interessen der verarmten Bevölkerungsschichten festgehalten, nicht zuletzt wohl deswegen, weil diese rechnerisch die Bevölkerungsmehrheit ausmachen. Dieses Festhalten bewirkte heftige innerparteiliche Kontroversen. Die diskutierte Alternative bestand im Wechsel zu einer klassen- bzw. schichtenübergreifenden Politik mit dem Ziel, das Wählerreservoir der politischen Mitte zu erreichen, um damit die Wahlchancen zu verbessern. Die Wahlniederlage in Nicaragua 1990 hatte ja gezeigt, daß sich die rechnerische Mehrheit keineswegs automatisch in eine elektorale umsetzt. Diese Option unterlag jedoch in beiden Parteien und konstitutierte sich fortan als eigene Partei. ${ }^{23}$

Wie schwierig jedoch eine Vertretung dieser Interessen im Rahmen des Parlaments ist, selbst wenn es sich um die der gesellschaftlichen Mehrheit handelt, zeigt sich an der Politik der FSLN seit ihrem Machtverlust 1990. Im parlamentarischen Verhandlungsprozeß kann sie als oppositionelle Partei die Interessen ihrer Klientel nicht oder nur geringfügig durchsetzen und ist zu Kompromissen gezwungen. Dadurch gerät sie wiederholt mit der Erwartungshaltung ihrer Wählerbasis in Konflikt und sieht sich dem Vorwurf des 'Verrats' ausgesetzt. Um sich ihrer sozialen Basis zu versichern, verleiht sie deren Anliegen durch außerparlamentarische Aktionen Nachdruck ${ }^{24}$, was sich jedoch als zweischneidig erweist, da diese sich gegen die parlamentarische Arbeit und damit gegen demokratisch zustandegekommene Entscheidungen richten. Mit solchen Aktionen entwertet die FSLN vor den Augen ihrer Basis diese Entscheidungen und unterminiert damit zugleich ihre eigene parlamentarische Arbeit. Enthält so einerseits der Versuch der außerparlamentarischen Durchsetzung dieser Interessen

reiche aus, erstreckt sich auf das Feld der Regierung: das Volk beeinflußt dann seine Regierung, bestimmt seine Regierung. In einer späteren Phase bedeutet Demokratie die Beteiligung der Arbeiter an der Leitung der Fabriken, Landwirtschaftsbetriebe, Kooperativen und sozialen Einrichtungen. Zusammenfassend gesagt ist Demokratie die Einmischung der Massen in alle Aspekte des gesellschaftlichen Lebens. (Barricada, 24. 8. 1980) Diese radikale Demokratieauffassung vertrat noch ein Jahr nach dem Sieg Humberto Ortega, der sandinistische Verteidigungsminister. Die nicaraguanische Revolution hatte ein Demokratiekonzept wieder auf die Tagesordnung gerufen, das in seiner Radikalität in Amerika seir der kubanischen Revolution nicht mehr in Angriff genommen worden war (Tangermann 1981).

23 In den Partido Democrático (PD), in El Salvador und in das Movimiento de Renovación Sandinista (MRS) in Nicaragua. Das von beiden anvisierte Wählerreservoir der Mitte ist sehr klein, dementsprechend auch die Wählerbasis beider Parteien.

24 Nach ihrer Wahlniederlage 1990 hat sie mehrfach zu diesem Mittel gegriffen und vor allem Managua tage-, manchmal wochenlang paralysiert (vgl. Rios 1995). 
ein hohes politisches Risiko für die FSLN als parlamentarischer Partei und erweist sich andererseits, daß dies innerhalb des parlamentarischen Rahmens ebenfalls nicht gelingt ${ }^{25}$, so offenbart sich ein Dilemma im Hinblick auf die Vertretung von Unterschichtsinteressen.

Im Rahmen parlamentarischer Politik scheint eine solche Interessenvertretung nur um den Preis des Verlusts der sozialen Basis möglich zü sein, während die außerparlamentarische Interessensartikulation eine Gefăhrdung für die parlamentarischen Handlungsmöglichkeiten darstellt. Noch genießen die großen Parteien der Linken aufgrund ihres zweigleisigen Handelns eine relativ breite Zustimmung in der Bevölkerung, doch steht außer Frage, daß die parlamentarische Option - allein schon aus elektoralen Gründen - die Oberhand gewinnen wird. Als Folge davon findet eine Repräsentation von Unterschichtsinteressen durch die Linksparteien zunehmend in nur noch symbolischer Weise statt.

In El Salvador ist das nicht anders: Nach ihrer Integration in das parlamentarische System haben die beiden Linksparteien FMLN und PD angesichts des Fortbestehens überkommener vordemokratischer Kräfte die Verteidigung der Regierbarkeit des demokratischen Regimes zur obersten Priorität deklariert, wie die übrigen Parteien auch. Für die beiden bislang stark an den Interessen ihrer Basis orientierten Parteien hat das zur Folge, daß statt der Verteidigung der Interessen ihrer sozialen Klientel nunmehr die Regierbarkeit des Landes zur Scheidelinie zwischen Freund und Feind wird. Der seit langem andauernde organisierte Protest gegen die Anpassungsund Stabilisierungspolitik der Regierung gilt ihnen daher nicht mehr als legitimes und parlamentarisch zi vertretendes Anliegen, sondern er

»wird von der Regierung und der Linken als wichtiger Baustein der Unregierbarkeit aufgefaßt. In dieser Phase der Befriedung (gemeint ist 1993-94, KDT) und Errichtung der Demokratie wird die auch früher übliche gewerkschaftliche Mobilisierung von jenen Linken und Rechten abgelehnt, die die neue soziale und politische Ordnung zu stützen versuchen« (Guido Béjar 1995: 166).

Im Maße, wie diese Parteien zunehmend auf die Vertretung besonderer Interessen verzichten und die Bindung an ihre traditionelle soziale Basis

25 Bobbio beschreibt sehr eindringlich die Unverträglichkeit von parlamentarischem Prinzip und der Repräsentation von Partikularinteressen (Bobbio 1988: 35-63).

26 Besonders deutlich ist die Aufgabe des klientelbezogenen zugunsten des Gesamtinteresses in der Abwehr jener sozialen Bewegungen festzustellen, die die Erfüllung der im Friedensabkommen - das ja die Grundlage der neuen Demokratie darstellt - vereinbarten staatlichen Leistungen wie Land, Kredite, Ausbildung oder Beschäftigung einfordern. Die Proteste nehmen häufig militante Formen an. »Regienung und Oppositionsparteien haben diese Formen sozialen Ausdrucks als Bausteine der Unregierbarkeit und sogar der Destabilisierung der neuen 'demokratischen Ordnung' im Aufbau betrachtet. Angesichts dieser Probleme verlangen sie nach höherer Effizienz der Polizei und nehmen zustimmend zur Kenntnis, daß sich die Streitkräfte (...) an Sicherheitsaufgaben beteiligen, wovon sie durch das Friedensabkommen gerade ausgeschlossen worden waren« (Guido Béjar 1995: 168). 
nur noch zu elektoralen Zwecken aktivieren, wandelt sich die ehemals postulierte Wahrnehmung der Unterschichtsinteressen in deren bloße symbolische Repräsentation. Für diese Schichten bedeutet das umgekehrt, daß sie die Repräsentation ihrer Interessen in der politische Sphäre, wie sie in den ersten Nachkriegsjahren in El Salvador von der FMLN und in den ersten Jahren nach dem Machtwechsel in Nicaragua von der FSLN wahrgenommen worden war, allmählich verlieren.

\section{Demokratien ohne Akzeptanz}

Bisher wurde die Frage der Demokratie in Mittelamerika anhand von Entstehungsfaktoren, Institutionen und Organisationen behandelt. Im folgenden Kapitel sollen die Meinungen und Haltungen der Menschen vorgestellt werden, von denen die Festigung demokratischer Verhältnisse letztlich abhängt. Damit sind im Unterschied zur Transitions- und Konsolidierungsforschung nicht die politischen Akteure und ihre mehr oder weniger demokratischen Auffassungen gemeint, sondern die Bevölkerung und ihre Haltung zu den demokratischen Regimes. Wie die folgenden Umfrageergebnisse belegen, ist das Vertrauen in die jungen Demokratien gering.

In Nicaragua hat sich dieses Vertrauen nach der Transformation des sandinistischen Systems in eine liberale Demokratie (1990) drastisch verringert (CID-Gallup 1992: 5, 11f). Ein Jahr nach dem Wechsel, 1991, war etwas über die Hälfte der Bevölkerung der Meinung, daß die Regierung ihre Interessen nicht vertrete; 1992 war dies bereits bei 72\% der Fall und zwei Drittel waren der Ansicht, daß auch die Parteien sich nicht um die Lösung der Probleme der Bevölkerung kümmerten (I.E.N. 1993: 8). Der repräsentative Aspekt der Demokratie stieß auf komplette Ablehnung: 98\% (!) der Befragten meinten, damit »wirkliche Demokratie« herrsche, müsse »das Volk zu wichtigen Entscheidungen konsultiert und an der Problemlösung beteiligt werden « (I.E.N. 1993: 15; Delgado 1994: 308). Von einer Akzeptanz des parlamentarischen Repräsentationsprinzip ist hier nichts zu erkennen.

In Guatemala bietet sich ein ähnliches Bild. Eine umfragengestützte Studie konstatiert eine »allgemeine Enttäuschung (...) im Hinblick auf die Instrumente der formalen Demokratie (und) noch mehr im Hinblick auf die politischen Institutionen und die staatlichen Organe.« Nur 11,4\% der Befragten sehen im Wählervotum einen geeigneten Mechanismus, die Probleme des Landes zu lösen (Gálvez 1995: 94ff). Von allen Ländem Mittelamerikas weist Guatemala die höchste Wahlabstinenz auf. Während in den anderen Ländern die jeweiligen Wahlbeteiligungen als ausreichende Legitimation angesehen werden, deutet die rund $70 \%$ ige Abstinenz in Guatemala darauf hin, daß hier das politische System nicht als legitime Interessenvertretung akzeptiert ist (Torres-Rivas 1991: 11; Jonas 1994). Diese Vermutung 
scheint besonders deshalb berechtigt, weil die Abstinenz trotz Regierungsund sogar Regimewechseln nicht abgenommen, sondern im Gegenteil von Wahl zu Wahl zugenommen hat. ${ }^{27}$

Die Daten für El Salvador sind nicht weniger ernüchternd. Auch hier betrachtet eine Mehrheit der Bürger Wahlen, die ja immerhin das einzige Instrument der Bürger zur politischen Einflußnahme darstellen, nicht als taugliches Instrument. 54,9\% meinen, diese verliefen nie oder nur selten sauber (Briones/ Ramos 1995: 279). Die Institutionen kommen nicht besser weg. Drei Fünftel der Bevölkerung $(60,2 \%)$ halten das Justizwesen für »immer oder häufig ungerecht « und etwa ebensoviele $(59,2 \%)$ lehnen Parteien $a b$, da sie ihre Interessen nicht vertreten sehen, und 43,6\% haben kein Vertrauen in das Parlament. Knapp die Hälfte $(47,1 \%)$ ist der Ansicht, die Regierung handele nie oder nur selten zum Wohle der Bevölkerung (ebd.: 256f).

Auf zwei Ergebnisse zu El Salvador sei noch hingewiesen. Zum einen, daß negative Auffassungen bei den besser Informierten weiter verbreitet sind als bei den weniger Informierten. Das verweist auf den erstaunlichen $\mathrm{Zu}-$ sammenhang, daß »die höchsten Indizes für Vertrauen an ebenso hohe Indizes von Unkenntnis über die entsprechenden Instanzen geknüpft (sind).« Zustimmung und Vertrauen in die demokratischen Institutionen entspringen also eher der Unkenntnis als demokratischer Überzeugung (ebd.: 262). Zum anderen besteht eine vergleichbare Korrelation in bezug auf die Schulbildung. Je niedriger diese ist, desto weniger interessieren sich die Befragten für Politik, haben keine Meinung oder äußern sich nicht. Kritik am politischen System ist in dieser Bevölkerungsgruppe, die zugleich über die geringsten Einkommen verfügt, am wenigsten ausgeprägt.

Bemerkenswert sind diese Untersuchungen auch deshalb, weil sie schichtspezifische Aussagen ermöglichen. Die untersten sozialen Gruppen sehen ihre bedrückendsten Probleme im wirtschaftlichen Bereich, gefolgt von sozialen Problemen, während politische an letzter Stelle folgen (ebd.: 266f). Das ist nicht überraschend, wenn man in Rechnung stellt, daß sich an der wirtschaftlichen Not nach dem Friedensabkommen nichts gebessert hat, sondern eher das Gegenteil eintrat. ${ }^{28}$

27 Die Wahlbeteiligung ist zwischen 1982 und 1991 von weniger als der Hälfte der Wahlbevölkerung auf weniger als ein Drittel zurückgegangen (Torres-Rivas 1991; 11, 14f). Bei den letzten Präsidentschaftswahlen lag die Wahlabstinenz (der Eingeschriebenen) in der ersten Runde (November 1995) bei 54\%, in der zweiten Runde (Januar 1996) bei 63\%. Nur rund 70\% der Berechtigten hatten sich einschreiben lassen (envio 167: 23f). Von allen staatlichen Institutionen genießt in Guatemala das Parlament die geringste Wertschätzung, gefolgt vom Justizwesen und den Parteien, während der Exekutive etwas mehr vertraut wird: Ganze 11,7\% geben an, Vertrauen in das Parlament zu haben, 13,1\% in das Justizwesen, nur 13,9\% vertrauen den politischen Parteien und 35,8\% der Regierung (Gálvez 1995: 102).

28 Trotz der Befriedung der Region und der Einführung der Demokratie mit dem Versprechen einer Besserung haben sich seither die sozioökonomischen Bedingungen, die in den 
Im einzigen Land mit vergleichsweise langer demokratischer Tradition, Costa Rica, wird zwar das Vorgehen der staatlichen Institutionen erstaunlicherweise ähnlich negativ beurteilt. Hier sind 59,3\% der Bevölkerung der Auffassung, die Parteien verträten nicht die Interessen der Bürger, 50,2\% sind der Meinung, das Parlament verdiene nicht das Vertrauen der Bevölkerung und $46 \%$ halten das Justizwesen für nicht gerecht. ${ }^{29}$ Doch gibt es einen entscheidenden Unterschied zu den übrigen Ländern Mittelamerikas in der Bewertung der Wahlen und der Exekutive: $82,5 \%$ halten die Wahlen für sauber und $55,8 \%$ meinen, die Regierung "arbeite für das Wohl des Landes«. Die Wahlbeteiligung liegt hier im Durchschnitt bei über $80 \%$ (Sojo 1995: 154ff).

Zusammenfassend läßt sich sagen, daß die Bevölkerung Mittelamerikas mit Ausnahme Costa Ricas - die Institutionen und Handlungen der demokratischen Regimes mehrheitlich nicht für vertrauenswürdig hält. Diese Ergebnisse beziehen sich auf das zentralstaatliche Institutionen- und Normengefüge und das Regierungshandeln. Die Bevölkerung sieht sich von diesen Instanzen nicht vertreten. Damit erweist sich die Repräsentation gesellschaftlicher Interessen in den demokratischen Institutionen als fehlgeschlagen. Die Funktionsweise der neuen Demokratien beruht offensichtlich weniger darauf, daß diese von demokratischen Individuen getragen als von mapathischen « geduldet werden.

Man kann annehmen, daß in Gesellschaften mit vergleichbaren sozioökonomischen Daten wie in Mittelamerika die Akzeptanz gleich welchen politischen Systems ebenfalls nicht sehr hoch sein wird. Hinzu kommt die geringe Neigung oder Fähigkeit der jungen Demokratien, die wirtschaftliche Lage der armen Bevölkerungsmehrheit zu verbessern, was eher die Enttäuschung als die Zustimmung gefördert hat. Ohne eine Verbesserung des Lebensniveaus ist eine demokratische Konsolidierung schwer vorstellbar. ${ }^{30}$

70er Jahren zu den revolutionären Auseinandersetzungen geführt haben, nicht verbessert, sondern im Gegenteil in mancher Hinsicht sogar verschlechtert, wie in der Einkommensverteilung (siehe Fußnote 17).

29 Hierin drückt sich eine neue Tendenz aus. Seit Beginn der 90er Jahre nehmen die negativen Urteile zu. Während 1988 die Frage, ob das Justizwesen Vertrauen verdiene, noch von $43 \%$ bejaht wird, gilt das 1994 nur noch für $27 \%$. Ähnlich die Auffassung über das Parlament: 1988 sind 28\% der Meinung, es »tauge nichts«. 1994 hat sich deren Anzah1 auf̂ $41 \%$ erhöht (Sojo 1995: 159).

30 Diese Aussage scheint von Meinungsumfragen insofern bestätigt zu werden, als selbst die tiefgreifenden politischen Veränderungen der letzten Jahre in der Hälfte der Bevölkerung offenbar keinen Eindruck hinterlassen haben. Die hier angeführten Daten stammen vom Anfang 1994, also zwei Jahre nach Beginn des Transformationsprozesses in El Salvador. Bereits drei Jahre zuvor, Anfang 1991 und damit ein Jahr vor dem Friedensabkommen, waren nahezu die gleichen Ergebnisse ermittelt worden (Briones/Ramos 1995: 258f). So ist zu vermuten, daß der mittlerweile vier Jahre währende Transformationsprozeß die Wahrnehmung des politischen Systems nicht verändert hat, wie auch eine Umfrage von Ende 1995 belegt (IUDOP- UCA 1995). Auf die 1994 gestellte Frage, ob in El Salvador 
Die Staaten Mittelamerikas haben das von der Modernisierungstheorie angegebene Einkommensniveau als Schwelle zur Möglichkeit von Demokratie inzwischen erreicht, doch als wichtiger für eine Stabilisierung der Demokratie erweisen sich Faktoren wie Verteilungsgerechtigkeit und die Schaffung institutioneller Repräsentationsmechanismen für die Unterschichtsinteressen. Die Umfragen haben gezeigt, daß die für entwickelte Demokratien typische auf Wahlen beschränkte Repräsentation die Bevölkerung nicht anspricht.

\section{Akzeptanz nicht-delegativer Repräsentationsformen}

Erst wenn wir die Ebene des staatlichen Institutionen- und Normengefüges und des Regierungshandelns verlassen und uns niedrigeren Politikebenen zuwenden, die statt symbolischen Handelns die Chance zur praktischen Einflußname versprechen, finden wir eine weniger ablehnende Haltung zur Politik vor. Der Wechsel von der zentralstaatlichen Politikebene zur dezentralen, lokalen oder gremialen beinhaltet zugleich eine andere Blickrichtung. Haben wir in der bisherigen Darstellung das Augenmerk auf die politischen Systeme und ihre Schwierigkeiten mit der demokratischen Konsolidierung gerichtet, sozusagen von oben geblickt, so schauen wir jetzt aus der anderen Richtung, nämlich von den politischen Handlungsformen her, in denen sich die Interessen besonders jenes Bevölkerungsteils ausdrücken, der sich von der zentralstaatlichen Politikebene ausgeschlossen fühlt.

Es ist insbesondere die Lokalpolitik, an die andere Politikerwartungen gerichtet werden. Eine ähnlich hohe Ablehnung wie die zentralen staatlichen Institutionen erfahren die lokalen nicht. Einige formelle staatliche Institutionen der Lokalpolitik erfreuen sich sogar einer deutlich höheren Wertschätzung. Diese Anerkennung wächst ihnen im Unterschied zu den zentralstaatlich orientierten Institutionen aus dem Grund zu, weil sie die Artikulation partikularer Interessen in nicht-delegativer Form zulassen. Sie bieten direkte Partizipationsmöglichkeiten an und ermöglichen die Erfahrung eigener Einflußnahme. Dasselbe gilt für informelle Institutionen der Zivilgesellschaft. In beiden Fällen handelt es sich um unmittelbare Politikformen im Unterschied zum symbolischen politischen Handeln.

Einer Studie über El Salvador zufolge ist die Partizipation an lokaler institutioneller Politik auf dem Land überraschenderweise hoch, während sie in größeren Gemeinden (ab 40.000 Einwohner) sehr gering ist. ${ }^{31}$ In den

Demokratie und wirkliche politische Freiheit bestehe oder ob alles so geblieben sei wie vor dem Friedensabkommen, antwortete knapp die Hälfte (46\%), es sei alles so wie früher. 42\% meinten, es existiere Demokratie (Briones/Ramos 1995: 245).

31 Die Untersuchung wurde 1994 im Auftrag des Büros für Wohnungs- und Stadtentwick- 
Städten ist demgegenüber eine höhere Beteiligung an der zentralstaatlichen Politik als auf dem Land ermittelt worden. Es zeigt sich hier im Vergleich mit den Haltungen zur zentralstaatlichen Politikebene ein umgekehrtes Resultat. Es läßt sich sagen, daß dort, wo die eigene Beteiligung die Chance auf Einflußnahme verspricht, d. h. vor allem auf dem Land und in kleinen Gemeinden, die Partizipation vergleichsweise hoch ist. Wo diese Chance nicht besteht, ist sie niedrig. Die Studie konstatiert eine enge Korrelation zwischen einer positiven Einschätzung der politischen Leistungen auf lokaler Ebene und einer positiven Haltung zur zentralstaatlichen Politik, die über die Partizipationserfahrung auf der lokalen Ebene vermittelt ist (Seligson/Córdoba 1995: 31) Das ist außerordentlich bemerkenswert, besagt es doch nichts anderes, als daß die positive oder negative Haltung zur zentralstaatlichen Politik und den staatlichen Instanzen von der Bewertung der Politik auf der lokalen Ebene geprägt ist.

Ein erheblicher Bevölkerungsteil, namentlich der Unterschichten, bildet sich seine Meinung gegenüber dem demokratischen Regime und zur Demokratie überhaupt auf lokaler Ebene, wobei dies um so mehr der Fall ist, wo die Partizipation an der lokalen Politik hoch ist. ${ }^{32}$ Hier zeigt sich eine spezifische Konstitutionsvariante politischer Haltungen, die bislang für El Salvador nachgewiesen ist, aber - mit der eventuellen Ausnahme Costa Ricas - so ähnlich auch in den anderen Ländern Mittelamerikas angenommen werden muß: Die Haltung zum politischen System insgesamt konstituiert

lung des USAID mit dem Ziel durchgeführt, die lokale Partizipation und die Haltung gegenüber den Lokalverwaltungen zu ermitteln. Die zugrundeliegenden Meinungsumfragen wurden in allen fünf Ländern Mittelamerikas angestellt. Unter Partizipation wird Teilnahme an Sitzungen der lokalen Regierungen verstanden. In einigen Ländern Mittelamerikas (Honduras, El Salvador, Nicaragua) finden mehrmals jährlich derartige Sitzungen als »cabildos abiertos« (offene Bürgerversammlungen der ganzen Gemeinde) statt. Die Beteiligung während zwölf Monaten (1994-95) lag im mittelamerikanischen Durchschnitt bei $11,3 \%$. In El Salvador lag sie deutlich höher (Wahljahr 1994). Während die Partizipation in vom Krieg nicht berührten Gebieten und in Städten allerdings sehr gering war, lag sie in ländlichen Gebieten erheblich höher und erreichte in den Gegenden, in denen die Unterstützung für die FMLN am höchsten ist, über $50 \%$ bei Frauen und mehr als zwei Drittel bei Männem (Seligson/Córdoba 1995: 23ff).

32 In der erwähnten Untersuchung wird keine Korrelation zwischen Partizipation als solcher und Unterstützung des politischen Systems hergestellt, aber eine solche zwischen Partizipation und positiver Leistung der Lokalregierung etabliert, wobei die Erfahrung von der Wirkung des eigenen Handelns von Bedeutung ist. In den Gegenden, in denen die Partizjpation an der lokalen Politik am höchsten ist - besonders Gebiete mit großer FMLNWählerbasis - fällt die Bewertung der Leistungen der Lokalregierung am positivsten aus. An dieser Korrelation von Zufriedenheit mit den Leistungen der lokalen Politik und Unterstützung des politischen Systems, Toleranz und demokratischen Normen zeigt sich, daß eine positive Haltung zum System bei einem Großteil der BürgerInnen dort wurzelt, wo ein unmittelbarer Einfluß auf politische Entscheidungen besteht. Die Partizipation ist in Mittelamerika nicht durchgängig gleich hoch. Sie ist auf lokaler Ebene in El Salvador höher als in den Nachbarländern (Seligson/Córdoba 1995: 26). 
sich nicht so sehr über die Bewertung der zentralstaatlichen politischen Sphäre, sondern nimmt den »Umweg« lokaler Politikerfahrung.

Hinter dieser für etablierte Demokratien eher untypischen Bedeutung der lokalen Politikebene kommt ein grundsätzliches Phänomen zum Vorschein: Anders als in konsolidierten Demokratien kommt in Mittelamerika der Ebene des symbolischen politischen Handelns kaum eine integrative Funktion zu, statt dessen konstituieren bei der Mehrheit der Bevölkerung direktere Formen politischer Erfahrung die Haltung zur zentralstaatlichen Politiksphäre und bilden damit das Fundament für die Akzeptanz oder Ablehnung der politischen Regimes.

Die direkte Politikform tendiert im Gegensatz zum atomisierenden Charakter symbolischer Politik zu kollektiven Haltungen. Im staatlich-institutionellen Rahmen erhält diese Politikform einen besonderen Charakter. Während nämlich andere direkte Formen partikularer Interessensartikulation, etwa gewerkschaftliche, im Kern darin bestehen, Einzelinteressen zu kollektiven Partikularinteressen zu bündeln, geschieht im Rahmen staatlicher Institutionen wie der Lokalpolitik etwas anderes: In der Vermittlung unterschiedlicher Positionen bilden sich überpartikulare Kompromisse und Haltungen heraus. ${ }^{33}$ Seligson und Córdoba haben entsprechende Ergebnisse ermittelt: In Gegenden mit hoher Partizipation besteht eine Korrelation mit Indizes hoher Toleranz und anderen demokratischen Eigenschaften (Seligson/Córdoba 1995: 28f).

Von den erwähnten Umfragen erscheinen mir besonders drei Aspekte von besonderer Bedeutung zu sein: Zunächst zeigen die Ergebnisse, in welch erstaunlich hohem Maße die zentralstaatlichen Institutionen und das Regierungshandeln der demokratischen Regimes noch zehn Jahre nach ihrer Einführung und fünf Jahre nach der Befriedung der Region in der Bevölkerung auf Ablehnung stoßen. Zum anderen zeigen sie eine deutlich andere politische Einstellung der Bürger, sobald es um die Politikebene geht, die diesen über symbolisches Handeln hinaus reale Partizipationschancen bietet. Dort ist besonders hervorzuheben, daß es diese partizipativen Erfahrungen sind, die erheblich zur Konstitution der Einstellung zur lokalen wie zur zentralstaatlichen Politikebene beitragen und die Entstehung demokratischer Haltungen fördern. Und schließlich die Schlußfolgerung, daß weder die zentralstaatlichen Institutionen und Normen, noch das wichtigste Instrument symbolischer Partizipation an politischen Entscheidungen, die Wahlen, eine nennenswerte integrative Funktion haben. Damit setzt sich

33 So auch Barber: Die öffentlichen Zwecke - wie er sie nennt - "werden buchstäblich im Akt der öfentlichen Partizipation geformt und durch gemeinsame Beratung wie gemeinsames Handeln geschaffen, wobei eine besondere Rolle spielt, daß sich der Gehalt und die Richtung von Interessen ändert, sobald sie partizipatorischen Prozessen dieser Art ausgesetzt sind (Barber 1994: 148). 
die soziale Spaltung der Gesellschaft unvermittelt auf der politischen Ebene in die Trennung von Gesellschaft und politischer Entscheidungssphäre fort. Die politische Integration der Gesellschaft scheint ohne Einbeziehung gesellschaftlich akzeptierter Repräsentations- und Partizipationsmechanismen nicht möglich zu sein.

\section{Partizipative Politikformen}

Ein breites Spektrum gesellschaftlicher Interessen ist in Mittelamerika nicht parlamentarisch, sondern außerparlamentarisch repräsentiert, so in Gewerkschaften, Kleinbauern- und Landarbeiterbewegungen, Unternehmer- und Selbständigenverbänden, Nachbarschafts-, Ökologie-, Frauenbewegungen und vielen mehr. Viele dieser Interessensorgane verfügen über eine erhebliche soziale Basis und üben einen starken Einfluß auf das politische Leben aus. Die Schwäche der Parteien und der Mangel an Vermittlungsmechanismen zwischen Staat und Gesellschaft ${ }^{34}$ und hat dazu geführt, daß diesen Organisationen traditionell die Repräsentation der kollektiven Interessen gegenüber der staatlichen Sphäre zugewachsen ist.

Der wesentliche Aspekt in diesem Zusammenhang ist, daß diese politischen Instanzen vorwiegend in nicht-delegativen Partizipations- und Repräsentationsformen agieren. Dabei ist zu betonen, daß Politik in Mittelamerika in erheblichem Maße in diesen unmittelbareren Formen stattfindet. An drei Beispielen sollen Wirkungsbereich und Grenzen partizipativer Politikformen vorgestellt werden. Zwei von ihnen sind im lokalpolitischen Bereich angesiedelt und das dritte entstammt dem Bereich kollektiver Interessenvertretungen.

Der erste Bereich, der sich für politisches Handeln in nicht-delegativen Formen anbietet, ist die schon erwähnte lokale Politik im staatlich-institutionellen Rahmen. Die Form politischer Partizipation ist hier weitgehend direkt. Sie bezieht sich im wesentlichen auf das Rathaus oder die Munizipalverwaltung und kanalisiert politische Interessen in die staatliche Sphäre.

34 Gewerkschaften und andere Organisationen erarbeiten seit längerem konkrete Vorschläge zur Behebung des »Mangels an wirksamen Vermittlungsinstanzen zwischen Gesellschaft und Staat (Rivera 1995: 246). Dabei geht es um institutionelle Strukturen, nachdem in den Vorjahren Erfahrungen mit ersten Verhandlungsforen in Form snationaler Forderungskataloge der Zivilgesellschaft (gemacht worden sind), so in Guatemala (Instancia Nacional de Consejo; Asamblea de Sectores Civiles) und in Honduras (Plataforma de Lucha, Coordinadora de Organizaciones Populares) « (Rivera 1995: 241). 1994 ist eine Instanz geschaffen worden, die eine mittelamerikanische Koordination der Zivilgesellschaften anstrebt, die »Iniciativa Civil para la Integración Centroamericana«, ICIC. In jedem Land nehmen Organisationen von Bauem- bis zu ökologischen Organisationen daran teil und koordinieren auf supranationaler Ebene ihre Forderungen und Vorschläge. Auch hier ist eines der Themen die Schaffung institutioneller Vermittlungsmechanismen $z w i-$ schen Gesellschaft und Staat (ICIC 1996). 
Neben dieser institutionellen Lokalpolitik bestehen in Mittelamerika zahlreiche weitere lokalpolitische Ansätze, die nicht vorrangig auf die institutionelle Einbringung von Interessen, sondern auf ein »empowerment« der Bevölkerung unabhängig von den staatlichen lokalen Instanzen abzielen. Dabei werden nach einer Verständigung über die drängendsten Probleme in den jeweiligen Gemeinden Handlungsoptionen entwickelt, deren potentielle Wirkung vor allem von der Zahl der Beteiligten abhängt. Derart auf Partizipation gegründete lokale Initiativen sind oftmals bald in der Lage, die institutionelle Lokalpolitik zu beeinflussen und diese auf eine stark partizipative Grundlage zu stellen. ${ }^{35}$

Die dritte Variante betrifft die sehr einflußreichen kollektiven Interessensorgane (ländliche Organisationen, Arbeiterorganisationen, soziale Bewegungen usw.), die die hauptsächlichen Artikulationsinstanzen der Unterschichten darstellen und unter denen die Zusammenschlüsse von Kleinund Mittelbauern das größte Gewicht haben. Diese Organe bündeln die vielen Einzel- zu Kollektivinteressen auf weitgehend partizipativer Grundlage. Allerdings sind weder die lokalen politischen Organe noch die Kollektivorganisationen auf den lokalen Raum beschränkt. Beide verfügen über regionale oder auch nationale Koordinationen. Auf der Ebene dieser höheren Repräsentationsstufen besteht die Möglichkeit direkter Partizipation nicht mehr und es werden delegative Politikformen notwendig. Die nichtdelegative Partizipationsform stößt hier schnell an ihre Grenzen und bleibt im wesentlichen auf die unteren organisatorischen Ebenen beschränkt. Sie erweist sich nicht nur als lokal, sondern auch inhaltlich beschränkt, da die Begrenzheit des lokalen Rahmens für die Behandlung darüber hinausgehender allgemeiner politischen Fragen nicht geeignet ist.

Obgleich also auch diese Organe partizipativer Politikformen nicht auf die delegative Repräsentation verzichten können, ist die Wirkung der delegativen Form dennoch eine andere bei der staatlichen symbolischen Repräsentation. Im Unterschied zum Delegationsprinzip des parlamentarischrepräsentativen Systems, wo der Delegierte die »Interessen (der Repräsentierten) nach eigenem Gutdünken interpretieren kann« (Bobbio 1988: 41), verfügt der Delegierte in diesen Organen zumeist nur über Vertretungsund in wesentlich geringerem Maße über Verhandlungsmacht, da er einem

35 In Mittelamerika kommt den Munizipien traditionell eine geringe institutionelle Bedeutung zu. Die politischen Parteien verfügen in der Regel über kein lokalpolitisches Konzept. Die zentralstaatliche Politik gegenüber den Munizipien trägt zumeist paternalistische Züge und wird weitgehend ohne Einbeziehung der Bevölkerung implementiert. Die systematische Bedeutung des Lokalen als Ort für empowerment hat erst die Ende der 80er Jahre enstandene Kommunalbewegung entdeckt. Empowerment-orientierte Bewegungen haben sich inzwischen in ganz Mittelamerika gebildet und verfügen über diverse Koordinationen (vgl. zu Nicaragua: Desarrollo Municipal 1996). 
stärkeren imperativen Druck unterliegt. Die delegative Repräsentation bewahrt auf diese Weise einen gewissen Anschein von Unmittelbarkeit.

Diejenigen Koordinationsebenen allerdings, in denen die Notwendigkeit der Verhandlung und des Kompromisses vorherrscht, vor allem die nationalen und supranationalen, unterliegen derselben Gefahr des Legitimationsverlusts, die weiter vorn anhand der Linksparteien geschildert wurde. Hier sind die Repräsentationswege zu lang und eine Kontrolle durch die Repräsentierten ist kaum noch möglich. Als um so notwendiger erweist sich das Instrument der Rechenschaftslegung und die innerorganisatorische Demokratie (Rivera 1995: 244ff). Tatsächlich ist in vielen dieser Organisationen und auch der Dachverbände eine Diskussion über interne Demokratie ausgebrochen.

Wie im Bereich der Lokalpolitik, so kann auch die partizipative Politikform der Interessenverbände nicht die parlamentarisch-repräsentative Politik ersetzen. Allerdings bildet sie die komplementäre Politikform, die als politische Artikulationsform vor allem der unteren Bevölkerungsschichten anzusehen ist.

\section{Literatur}

Alarcón, Victor, Guillermo O'Donnell, Adam Przeworski (1994): Democracia sustentable. In: Espacios, Nr. 1, julio-septiembre. San José. (FLACSO Costa Rica). S. 104-107.

Barber, Benjamin (1994): Starke Demokratie. Über die Teilhabe am Politischen. Berlin (Rotbuch).

Bastos, Santiago; Manuela Camus (1995): Abriendo caminos. Las organizaciones mayas desde el Nobel hasta el Acuerdo de Derechos Indigenas. Guatemala (FLACSO).

Bendel, Petra; Michael Krennerich (1996): Zentralamerika: Die schwierige Institutionalisierung der Demokratie, in: Merkel et al. (1996), S. 315-340.

Berger, Johannes (1996a): Modernisierung und Modernisierungstheorie, in: Leviathan, H. 1 (März), Jg. 24, Wiesbaden. S. 8-12.

Berger, Johannes (1996b): Was behauptet die Modernisierungstheorie wirklich - und was wird ihr bloß unterstellt?, in: Leviathan, H. 1 (März), Jg. 24, Wiesbaden. S. 45-62.

Beyme, Klaus von (1994): Ansätze zu einer Theorie der Transformation der ex-sozialistischen Länder Osteuropas, in: Merkel (1994), S. 141-171.

Bobbio, Norberto (1988): Repräsentative Demokratie und direkte Demokratie, in: ders., Die Zukunft der Demokratie. Berlin. S. 35-63.

Bonner, Raymond (1984): Weakness and Deceit. U.S. Policy and El Salvador. New York (Times).

Bos, Ellen (1994): Die Rolle von Eliten und kollektiven Akteuren in Transitionsprozessen, in: Merkel (1994.), S. 81-109.

Briones, Carlos; Carlos G. Ramos (1995): La gobernabilidad en Centroamérica (3): economia, gobemabilidad y democracia en El Salvador. San Salvador (FLACSO).

CID-Gallup (1992): Opinión Pública, Nicaragua No. 7, Noviembre. Managua.

Dahl, Robert A. (1992): La democracia y sus críticos [1989]. Barcelona. (Paidós).

Delgado Romero, Rodolfo (1994): Nicaragua: gestión estatal, sistema de partidos y democracia local. In: Maihold, Günther; Manuel Carballo Quintana (comp.) (1994): ¿Qué será de Centroamérica?: Gobernabilidad, legitimidad electoral y sociedad civil. San José (Friedrich Ebert Stiftung; CEDAL), S. 293-314. 
Desarrollo Municipal (1996): Primer informe semestral. Enero-Junio 1996 (Projektbericht an Buntstift, Göttingen). Managua.

Diamond, Larry (1992): Economic Development and Democracy Reconsidered, in: Larry Diamond und G. Marks (eds.), Reconsidering Democracy, London: Sage, S. 93-137.

Diamond, Larry; Juan J. Linz; Seymour Martin Lipset (1990): Politics in Developing Countries. Comparing Experiences with Democray. Boulder (Lynne Rienner).

Di Palma, Giuseppe (1990): To Craft Democracies. An Essay on Democratic Transitions. Berkeley (University of California Press).

envio. Revista mensual de la Universidad Centroamericana (UCA) (1996). 15. Jahr, Nr. 167 (Januar-Februar). Managua.

FLACSO (1995): Centroamérica en cifras 1980-1992. San José.

Flora, Jan L.; Edelberto Torres-Rivas (1989): Sociology of Developing Countries; Historical Bases of Insurgency in Central America. In: Jan L. Flora and Edelberto Torres-Rivas (Hrsg.), Sociology of "Developing Countries". Central America. Houndmills and London (Macmillan). S. 32-55.

Gálvez Borrell, Víctor (1995): La gobernabilidad en Centroamérica (2): sectores populares y gobernabilidad precaria en Guatemala. Guatemala (FLACSO).

Guido Béjar, Rafael (1995): Reflexiones sobre los movimientos sociales, la sociedad civil y los partidos politicos en El Salvador de post guerra, in: ders./Stefan Roggenbuck (eds.), Sociedad participativa en El Salvador. San Salvador (Konrad Adenauer-Stiftung; UCA). S. 155-177.

Habermas, Jürgen (1977): Zum Begriff der politischen Beteiligung [1958], in: Kultur und Kritik. Frankfurt/M. (Suhrkamp).

ICIC (Iniciativa Civil para la Integración Centroamericana) (1996): Documento de consulta para el proceso previo a la segunda asamblea general de la ICIC. o.O. .

I.E.N. (Instituto de Estudios Nicaragüenses) (1993): La problemática de la gobernabilidad en Nicaragua. Informe de investigación sobre la opinión pública nacional. Resumen ejecutivo de la investigación. Managua. 25 de marzo.

IUDOP-UCA (1995): Encuesta sobre el sistema politico salvadoreño.Consulta de opinión pública de octubre de 1995 (hier zit. n. Proceso. Informativo Semanal (1995). San Salvador (UCA), 16. Jahr, Nr. 690, 13. Dezember.

Jonas, Susanne (1994): Guatemala. El problema democrático. In: Nueva Sociedad, Nr. 130, marzo-abril, Caracas. S. 15-23.

Karl, Terry Lynn (1990): Dilemmas of Democratization in Latin America, in: Comparative Politics, Vol. 23, № 1. S. 1-21.

Karl, Terry Lynn; Philippe C. Schmitter (1991): Modes of Transition in Latin America, Southern and Eastem Europe, in: International Social Science Journal, Nr. 128. S. 269-284.

Lechner, Norbert (1993): Modernización y modernidad: la búsqueda de ciudadanía. En: Centro de Estudios Sociológicos (ed.), Modemización económica, democracia politica y democracia social. México D.F. (Colegio de México). S. 63-75.

Linz, Juan J.; Alfred Stepan (1996): Toward Consolidated Democracies, in: Journal of Democracy. Vol. 7, №2 (April). Baltimore. S. 14-33.

Lipset, Seymour Martin (1993): El hombre politico. Las bases sociales de la política [Orig.: Political Man. 1959]. México D.F. (Rei).

Lipset, Seymour Martin; Kyong-Ryung Seong; John Charles Torres (1994): Análisis comparado de los requisitos sociales de la democracia [1993]. In: Condiciones sociales de la democracia. Cuaderno de ciencias sociales, no. 71. San José. (FLACSO). S. 9-58.

Lungo, Mario (1993): Los obstáculos a la democratización en El Salvador, in: El Salvador en construcción, Nr. 11, agosto. San Salvador. S. 21-32.

Maihold, Günther (1994): Representación política y sociedad civil en Centroamérica. In: Maihold, Günther; Manuel Carballo Quintana (comp.), ¿Qué será de Centroamérica?: Gobernabilidad, legitimidad electoral y sociedad civil. San José (Friedrich Ebert Stiftung; CEDAL). S. 203-223. 
Menjivar, Rafael; Juan Diego Trejos (1992): La pobreza en América Central. San José (FLACSO), 2a. ed..

Merkel, Wolfgang (Hrsg.) (1994): Systemwechsel 1. Theorien, Ansätze und Konzeptionen. Opladen.

Merkel, Wolfgang et al. (Hrsg.) (1996): Systemwechsel 2. Die Institutionalisierung der Demokratie. Opladen.

Merkl, Peter H. (1994): Cuáles son las democracias de hoy? [1993] In: Condiciones sociales de la democracia. Cuaderno de ciencias sociales, no. 71. San José. (FLACSO). S. 59-90.

O'Donnell, Guillermo (1993): Estado, democratización y ciudadanía, in: Nueva Sociedad, Nr. 128 , noviembre-diciembre. Caracas. S. 62-87.

O’Donnell, Guillermo (1994): ¿Democracia delegativa? [1991]. In: Barba, Jaime (comp.), La democracia hoy. San Salvador (Istmo). S. 11-32.

O'Donnell, Guillermo (1996): Illusions about Consolidation, in: Journal of Democracy. Vol. 7, № 2 (April). Baltimore. S. 34-51.

Offe, Claus (1980): Konkurrenzpartei und kollektive politische Identität. In: Roland Roth (Hg.), Parlamentarisches Ritual und politische Alternativen. Frankfurt (Campus). S. 26-42.

Przeworski, Adam (1991): Democracy and the Market. Cambridge (University Press).

Ríos, Ivana (1995): Participación de los sectores populares en Nicaragua: cambios actuales 1990-1994, in: Tangermann (1995), S. 313-352.

Rivera, Rolando (1995): Concertación social e integración regional: ¿una nueva forma de participación social?, in: Tangermann (1995), S. 207-261.

Rojas Bolaños, Manuel (1994): Las relaciones partido - gobierno: el caso de Costa Rica. Ponencia IX Congreso centroamericano de Sociologia. San Salvador, 18.-22. 7. mimeo.

Rojas Bolaños, Manuel (1995): Consolidar la democracia en Centroamérica: una ardua tarea, in: Tangermann (1995), S. 99-1 55.

Samour, Héctor (1994): Marco teórico para la construcción de un orden democrático en El Salvador. In: Estudios Centroamericanos, Nr. 543-544, enero-febrero. San Salvador. (UCA). S. 33-55.

Schmitter, Philippe C. (1993): La consolidación de la democracia y la representación de los grupos sociales [1992]. In: Revista Mexicana de Sociologia, año LV, no. 3, julioseptiembre. México D.F. (UNAM). S. 3-30.

Schumpeter, Joseph A. (1993): Kapitalismus, Sozialismus und Demokratie [1950]. Tübingen und Basel.

Seligson, Mitchell A.; Ricardo Córdova (1995): Gobierno local y democracia en El Salvador. In: Espacios, Nr. 4, abril-junio. San José (Friedrich Ebert Stifung; FLACSO; CEDAL). S. 21-33.

Sojo, Carlos (1995): La gobernabilidad en Centroamérica (4): la sociedad después del ajuste. Demandas sociales, reforma económica y gobernabilidad en Costa Rica. San José (FLACSO).

Tangermann, Klaus-Dieter (1981): Der Aufbau der »Sandinistischen Demokratie «, in: Veronika Bennholdt-Thomsen et al. (Hg.), Lateinamerika. Analysen und Berichte 5. Berlin (Olle \& Wolter). S. 185-203.

Tangermann, Klaus-Dieter, Ríos, Ivana (coords.) (1994): Alternativas campesinas. Modernización en el agro y movimiento campesino en Centroamérica. Managua (CRIES-Latino Editores).

Tangermann, Klaus-Dieter (comp.) (1995): Ilusiones y dilemas. La democracia en Centroamérica. San José (FLACSO, Buntstift).

Tetzlaff, Rainer (1992): Die blaue Blume der Demokratie - Thesen zur Übertragbarkeit eines westlichen Modells. In: Der Überblick, Nr. 3. Hamburg. S. 11-14.

Torres-Rivas, Edelberto (1990a): Democracias de baja intensidad [1989]. In: Edelberto Torres-Rivas, El sistema político y la transición en Centroamérica. Cuadernos de ciencias sociales, no. 36. San José (FLACSO). S. 35-51. 
Torres-Rivas, Edelberto (1990b): La transición autoritaria hacia la democracia. In: Edelberto Torres-Rivas, El sistema político y la transición en Centroamérica. Cuadernos de ciencias sociales, no. 36. San José (FLACSO). S. 53-75.

Torres-Rivas, Edelberto (1991): Imágenes, siluetas, formas en las elecciones centroamericanas: las lecciones de la década. In: Polémica, no. 14-15, San José. (FLACSO). S. 2-21.

Torres-Rivas, Edelberto (1992): La democracia latinoamericana en la fragua. In: Edelberto Torres-Rivas, El tamaño de nuestra democracia. San Salvador. (FLACSO; istmo). S. 131-153.

Torres-Rivas, Edelberto (1994): La gobernabilidad centroamericana en los noventa. (Consideraciones sobre las posibilidades democráticas en la postguerra). In: Maihold, Günther; Manuel Carballo Quintana (comp.), ¿Qué será de Centroamérica?: Gobernabilidad, legitimidad electoral y sociedad civil. San José (Friedrich Ebert Stiftung; CEDAL). S. 53-69.

Vilas, Carlos M. (1996): Prospects for Democratisation in a Post-Revolutionary Setting: Central America, in: Journal of Latin American Studies, 28. (Cambridge Univ. Press) S. 461-503.

\section{PROKLA 106 (März 1997): Klassen und Politik in Deutschland}

In den gewerkschaftlichen Debatten gelten Linke vielfach als ewiggestrige »Traditionalisten «, diejenigen, die nicht nur auf Kooperation mit dem Kapital, sondern gleich auf Co-Management setze dagegen als entschiedene »Modernisierer «. Bei den Unternehmern wird nicht nur der Flächentarifvertrag in Frage gestellt, sondern die Existenz der eigenen Verbände gleich mit. Das Verhältnis von Lohnarbeit und Kapital scheint sich in Deutschland neu zu strukturieren. Ist dies alles nur eine Anpassung an die europäisch-nordamerikanische Normalität oder drükken sich hier grundsätzliche Veränderungen aus? In Deutschland nehmen die Geldvermögen nicht nur immer schneller zu, die Politik unterwirft sich auch immer stärker der Logik ihrer Verwertung. Die Kehrseite des privaten Reichtums ist die öffentliche Armut. Welche Rolle kann unter diesen Umständen noch ein Sozialstaat spielen? Leistet sich jetzt auch der entwickelte deutsche Kapitalismus die langfristige Exklusion einer »Underclass «? 